\title{
Safer fluid prescribing at North Bristol Trust: Bringing practice in line with NICE Guidance with a redesign of the fluid prescription chart
}

\author{
Johanna Andersson, Thomas Bull, Daniel Paul, Emily Reynolds, Aimi Nishimura, Jessica Lloyd, Amanda Jetley, Mari Davies, Helen \\ Eisenhauer, Daniel Maggs, Sorcha Clapham, Kaushali Trivedi, Robert Hair, Benjamin Plumb, Benjamin Plumb \\ North Bristol Trust
}

\begin{abstract}
The National Institute of Clinical Excellence (NICE) released new fluid guidelines following data suggesting $20 \%$ of patients receiving fluids suffer adversely (2013). This quality improvement group assessed fluid prescribing in a tertiary teaching centre and introduced a new fluidprescribing chart to align practice with NICE recommendations.
\end{abstract}

Notes and corresponding fluid prescription charts were reviewed for evidence of (1) indication, (2) co-morbidities, and (3) further management as surrogate markers of safe prescribing in accordance with NICE. Overall, the data showed practice fell short and prompted a redesign of the Trust fluid prescription chart. Three different variations of the chart were issued consecutively using a PDSA method (plan, do, study, act) over a 6-month period. They all included indication, co-morbidities and further management plans as constant design features. Suggestions from interested parties were incorporated and an educational programme was implemented to promote awareness.

Prior to our intervention, an indication for fluids was documented in $26 \%$ of notes, it took an average of 4.6 minutes to find co-morbidities, and further management plans were rarely documented. Following the new prescription chart, an indication was recorded in $72 \%$ of cases, comorbidities noted on $63 \%$ of charts with $93.1 \%$ accuracy, and further management documented in $100 \%$ of cases.

The new fluid prescription chart encourages prescribers to incorporate NICE recommendations when prescribing fluids. The new fluid prescription design has since been rolled out Trust wide.

\section{Problem}

As highlighted by the new 2013 NICE guidelines on intravenous fluids, assessment and prescription of fluids in a hospital setting is challenging, and is often left to the junior doctors in the team (1). Anecdotal evidence from foundation year 1 doctors at North Bristol Trust (2013-14) supported this, highlighting particular problems prescribing fluids for patients during on-call shifts. Often, juniors reported a lack of clear indication for fluids, poor documentation of plans in notes for further fluid management, and difficulty finding relevant information for safe prescribing effectively and efficiently.

Our QI group suspected that these poor conditions for fluid prescribing were contributing to incorrect, inadequate, delayed, and over-prescribed intravenous fluids, leading to increased morbidity and mortality in patients.

\section{Background}

'The National Confidential Enquiry into Peri-operative Deaths, 1999 suggested a significant proportion of patients were dying as a result of inappropriate fluid prescription, prompting the report to conclude that it should be given the same gravitas as drug prescription (2). This is compounded by the recent NICE fluid guidelines, where it is suggested 1 in 5 patients who receive intravenous fluids suffer an adverse outcome (1). With most fluid prescriptions being completed by junior doctors, further research is desperately needed. There is evidence in 'The BMJ Quality Improvement Report,' that there is increasing awareness of this, with projects promoting NICE fluid prescribing with lanyard cards (3), labels with cautions attached to fluid charts (4), and stickers on drug charts to prompt consideration of indication and cautions when prescribing fluids (5). With this project we hope to improve fluid prescribing at our Trust, but also contribute to existing quality improvement projects and inspire others to initiate similar strategies in Trusts across the Nation.

\section{Baseline measurement}

This project took place at North Bristol Trust (NBT), a large tertiary teaching hospital, within a one-year period. Three measures were recorded to assess the extent to which NBT practice was in accordance with NICE guidelines:

1. Time taken to find an indication for fluid prescription

2. Whether a fluid management plan was documented in the notes, and

3. Time taken to identify co-morbidities

The results indicated that NICE recommendations were not being fulfilled. In 4 of the 15 patients (26.7\%) an indication was given, and of those identified, only 3 were found in less than 2 minutes.

Documentation of an intravenous management plan was present in 4 of 15 patients $(26.7 \%)$, and it took on average 3.5 minutes to find 
relevant co-morbidities in notes. This supported the anecdotal evidence provided by junior doctors at NBT.

\section{Design}

It was felt that the introduction of a new fluid prescription chart would be a sustainable way to implement change. Three PDSA (plan, do, study, act) cycles were carried out over a six month period at NBT, in which three variants of one basic fluid chart design evolved in response to data collected.

To give an idea of the basic design:

- A caution box at the top of the chart was added for doctors to be able to document and quickly gain an over-view of relevant co-morbidites.

- A column at the start of the chart was added for indication, to prompt prescribers to consider carefully why they were giving fluids.

- A column towards the end of chart was added for 'what to do once fluid bag runs out,' as a proxy for documentation of a further fluid management plan. This was meant to aid communication between day/on call team, but also to clarify the plan for nurses, which the pilot study suggested were often the initiators of fluid prescription reviews.

- The chart was designed in landscape format rather than portrait (as previously) in order to minimize number of rows for fluid prescription. It was felt this would prompt consideration of whether a new chart (ie more fluid) needed to be started.

- More space for the prescribers signature was given and a diagonal line added to prompt initials/stamps. This was to facilitate better identification of who the prescriber was to limit bleeps to practitioners who did not know that patient.

Whilst the additional ink, and subsequent cost of printing a more complex chart was considered, it was felt the Trust would benefit from safer fluid prescription resulting in reduced cost associated with morbidity and mortality. This QI group also speculated that increased consideration with regards to fluid prescription would reduce the number of charts printed. It is this groups intention to establish this hypothesis with retrospective analysis of the number of charts produced during the premier year of the new chart versus number charts printed the year before inducing change.

\section{Strategy}

PDSA Cycle 1: Introduction of the new fluid prescription chart on the surgical assessment unit and four medical wards. As part of this process, the old chart was temporarily removed. Head of nursing was informed on the day of roll, a brief teaching session was given to the relevant doctors on the ward and posters displayed. Chart 1 : Included a free box for indication on the chart, a fluid management column with options to circle one of three plans (continue fluids/stop fluids/ask a doctor to review) for each prescription, and a cautions box at the top of the chart including tick boxes for liver, renal, and heart failure, with options to tick 'other,' to specify an unlisted co- morbidity and 'none,' to indicate no medical condition.

PDSA Cycle 2: Following collection and analysis of data from the PDSA cycle 1, we introduced the second version of the fluid prescription chart onto the same wards. This chart was accompanied by a formal teaching session at the monthly foundation year 1 trust teaching, a further poster campaign that was extended to all clinical areas. Informal feedback from nursing staff and other members of the team were collected and taking into consideration during this process. This chart had three options for prescribers to choose from in the 'indications' column -

resuscitation, maintenance, or replacement in accordance with NICE terminology. We added options to specify more information regarding the failures in the cautions box and made no change to the 'further management' column.

PDSA Cycle 3: We found the previous changes worsened outcome, so we reverted to the free text indications column, educated juniors about how to fill out our cautions box, and changed the three options in the 'further management column' to tick box style rather than circling. This was accompanied by all of the essential advertising and education as the previous charts. In addition, we took the chart to all the required hospital committees to gain approval for its use trust wide.

See supplementary file: ds4358.docx - "PDSA Cycles"

\section{Post-measurement}

Ultimately, chart 3 showed a vast improvement in documentation of all 3 measures compared to the original trust fluid prescription chart. Indication for intravenous fluids was given in $72 \%$ of cases compared to $26.7 \%$, management plans were established for $100 \%$ of patients compared to almost none being given in our pilot study, and documentation of relevant co-morbidities improved from $0 \%$ to $63.6 \%$.

See supplementary file: ds3757.docx - "Run Chart: Changes in Documentation of Indication, Management, and Co-morbidities in each PDSA cycle."

\section{Lessons and limitations}

During this process we learnt a lot about the natural evolution of QI projects. 1) For any change, it is important to identify key measures that you want to employ. Without this it is difficult to make comparisons and to show real change. As a group we spent a long time deciding on indication, co-morbidities, and management plans. 2) It is vital to involve interested parties early on in the process. The more engaged relevant groups are in embryonic formulations of a change, the better the change ultimately grows to be. It also has a greater likelihood of being successful. 3) During the process it is extremely important to have regular group gatherings to ensure all team members are aware of targets for the week/month/cycle. By doing this, a unified approach is facilitated that strengthens the message being spread across the Trust, and changes made. 


\section{BMJ Quality Improvement Reports}

\section{Conclusion}

This project started by responding to anecdotal evidence at NBT, which suggested junior doctors were struggling to prescribe fluids in on-call settings. Research on the topic indicated there was a lack of audits/QI evidence for fluid prescription outside specialist settings (theatre, anaesthesia, ICU). In addition, recent NICE guidelines implied significant mortality and morbidity associated with poor fluid prescribing in the UK.

This group set out to improve ease and safety of fluid prescription at NBT by implementing a new chart to include measures that would align practice with NICE recommendations, thereby reducing fluid associated mortality and morbidity. During a 6 month period a significant improvement to documentation of indication for fluids, further fluid management plans, and comorbidities of patients was made as a result of 3 PDSA cycles. The improvement was so compelling that with support of interested parties the chart was rolled out Trust wide. However, to date it is difficult to establish to what extent the new chart has reduced mortality and morbidity in patients at NBT. We can only hope that by aligning NBT fluid prescription with NICE recommendations, we are by default improving patient safety.

\section{References}

1. National Institute for Health and Care Excellence (2013) Intravenous fluid therapy in adults in hospital [174] London: NICE; Perioperative Deaths London: NCEPOD

2. National Confidential Enquiry into Patient Outcome and Death (1999) Report on Perioperative Deaths London: NCEPOD;

3. Sansom LT, Duggleby L. Intravenous fluid prescribing: Improving prescribing practices and documentation in line with NICE CG174 guidance. BMJ Qual Improv Report 2014;3: doi:10.1136/bmjquality.u205899.w2409

4. Hartridge-Lambert S, Moore L, Walker O, Wilkinson D. Improving out-of-hours intravenous fluid prescribing for junior doctors: a prescription label. BMJ Qual Improv Report 2013;2:1 u632.w638 doi:10.1136/bmjquality.u632.w638.

5. Fehmi J, Carpenter A, Townsend M, Sheppeard R, Gannon E, Hewitson L, Auerbach N, Thomas K. Out of hours intravenous fluid therapy: a prompt to guide prescribing. BMJ Qual Improv Report 2015;4:1 u204010.w3139 doi:10.1136/bmjquality.u204010.w3139

\section{Declaration of interests}

Nothing to declare

\section{Acknowledgements}

Dr Seema Srivastava, Dr Katherine Finucane, Dr Kate Nickell, Dr Murli Krishna, Dilesh Khandhia, Stephen Taylor, NBT Audit

Department. 